\title{
THE DESIGN OF TWO-DIMENSIONAL AEROFOILS WITH MIXED BOUNDARY CONDITIONS*
}

\author{
BY \\ L. C. WOODS \\ Sydney University, Australia
}

Summary. A method is given of designing two-dimensional symmetrical aerofoils in subsonic compressible flow when the boundary conditions are mixed. The aerofoil surface is divided into three parts such that the shape is prescribed in the first and third and the pressure distribution is specified in the second. The method has the advantage over the usual method of aerofoil design (pressure distribution given over the whole aerofoil surface) of giving the designer direct control of the nose shape and the trailing edge angle.

1. Introduction. Lighthill's paper [1] on aerofoil design in incompressible flow and a subsequent extension of the method by the author [2] to compressible flow deal with the case when the pressure distribution is specified over the whole of the aerofoil. Now while the designer wants to control his pressure distribution over the front half or twothirds of the chord he is usually more concerned about the shape of the aerofoil towards the trailing edge than the corresponding theoretical pressure distribution, which in any case is not attained in practice because of the action of viscosity. Indeed a number of aerofoils which have been designed by Lighthill's method have been subsequently improved by modifying the shape near the trailing edge. It thus appears that a theory which permits the specification of the pressure distribution over the front part of the chord and the shape over the rear part would be of some practical value. The theory given below is a little more general than this as it also allows the shape of the aerofoil nose to be specified. The chord is thus divided into three parts in which shape, pressure and shape are specified respectively. The design problem is the determination of the shape of the middle section to give the specified pressure distribution.

The theory is developed for a symmetrical aerofoil at zero incidence moving at subsonic speeds. It is exact for both an incompressible gas and a 'Karman-Tsien tangent gas', and hence for a real gas it is an approximate theory, applicable to most of the subsonic Mach number range. The basic mathematical theory is as follows.

The equipotentials $(\phi=$ constant $)$ and streamlines $(\psi=$ constant $)$ of an inviscid compressible flow are defined by

$$
d \phi=q d n, \quad d \psi=\frac{\rho}{\rho_{0}} q d n,
$$

where $\rho, \rho_{0}$ are the local and stagnation densities respectively; $n, s$ are distances measured normal to and along a streamline respectively; and $q$ is the velocity magnitude. Values in the undisturbed stream at infinity will be denoted by a subscript ' $\infty$ '. The independent variables of the theory are $\theta$, the flow direction measured from the direction of flow at infinity (i.e. $\theta_{\infty}=0$ ), and $\Omega$, defined by

$$
\Omega=-\int_{U}^{a} \frac{\beta}{q} d q,
$$

where $U=q_{\infty}, \beta=\left(1-M^{2}\right)^{1 / 2}$, and $M$ is the local Mach number.

*Received May 17, 1954. This work was performed while the author was a member of the New Zealand Scientific Defense Corps, attached to the Aerodynamics Division of the N.P.L., England. 
Consider an imaginary gas for which the number $m$ defined by

$$
m=\beta \rho_{0} / \rho,
$$

is constant, and equal to the value it would have for an ideal gas in the undisturbed stream [6]. Such a gas can be shown to have two important properties. Firstly the complex function,

$$
f=\Omega+i \theta,
$$

is easily shown [3] to be an analytic function of

$$
w=\phi+i m_{\infty} \psi,
$$

where $m_{\infty}$ denotes the value of $m$ at infinity in an ideal gas. Secondly, if $p$ is the gas pressure, the $(p, 1 / \rho)$ curve is a straight line tangential to the curve for an ideal gas at $\left(p_{\infty}, 1 / \rho_{\infty}\right)$. This second property is quite well-known, but it is convenient to outline the proof briefly here.

From Eq. (3) it follows that the imaginary gas is defined by

$$
\frac{a^{2}-q^{2}}{a^{2} \rho^{2}}=\left(\frac{\beta_{\infty}}{\rho_{\infty}}\right)^{2}
$$

where $a$ is the local speed of sound. The tangent to the adiabatic curve of an ideal gas is $d p / d \rho=a^{2}$, so replacing $a^{2}$ in Eq. (6) by this gradient ensures that the adiabatic curves of the imaginary and ideal gases can touch. From Eq. (6) and Bernoulli's equation, namely

$$
\frac{d p}{\rho}+q d q=0
$$

it then follows that

$$
\rho^{2} \frac{d p}{d \rho}=a_{\infty}^{2} \rho_{\infty}^{2}
$$

or

$$
\rho^{2} a^{2}=a_{\infty}^{2} \rho_{\infty}^{2} .
$$

Further integration yields

$$
p-p_{\infty}=-a_{\infty}^{2} \rho_{\infty}^{2}\left(\frac{1}{\rho}-\frac{1}{\rho_{\infty}}\right),
$$

which is a straight line in the $(p, 1 / \rho)$ diagram, tangential at $\left(p_{\infty}, 1 / \rho_{\infty}\right)$ to the ideal gas curve.

The relation between $\Omega$ and $q$ is required in the following theory. From (6) and (7) it follows that

$$
a^{2}-q^{2}=a_{\infty}^{2}-q_{\infty}^{2}
$$

and hence

$$
a \beta=a_{\infty} \beta_{\infty} .
$$

Thus from $q=a M$,

$$
\frac{q}{U}=\frac{M}{\beta} \frac{\beta_{\infty}}{M_{\infty}}=\frac{\left(1-\beta^{2}\right)^{1 / 2}}{\beta} \frac{\beta_{\infty}}{M_{\infty}} .
$$


(It should be noticed from Eq. (8) that a tangent gas can never have supersonic patches embedded in it.) From Eqs. (2) and (8) it follows after some algebra that

$$
\begin{gathered}
\frac{g}{U}=\sinh \epsilon \operatorname{cosech}(\Omega+\epsilon), \\
\epsilon=\sinh ^{-1}\left(\beta_{\infty} / M_{\infty}\right) .
\end{gathered}
$$

If the flow is incompressible, $\Omega=\log (U / q), m_{\infty}=1$, and

$$
f=\log \left(\frac{U}{q} e^{i \theta}\right)=\log \left(U \frac{d z}{d w}\right),
$$

where $z=x+i y$ is the physical plane. In incompressible flow $z=z(w)$, and so (11) yields immediately that $f=f(w)$.

Since $f$ is an analytic function it satisfies

$$
\nabla^{2} f=0,
$$

in the $w$-plane, or in any plane derived from the $w$-plane by a conformal transformation. The solution of this equation with the appropriate boundary conditions follows in the next section.

2. Mathematical theory. Figure 1 shows half of a symmetrical aerofoil at zero incidence. The shape of the aerofoil is assumed known in the intervals $B C$ and $D E$, while

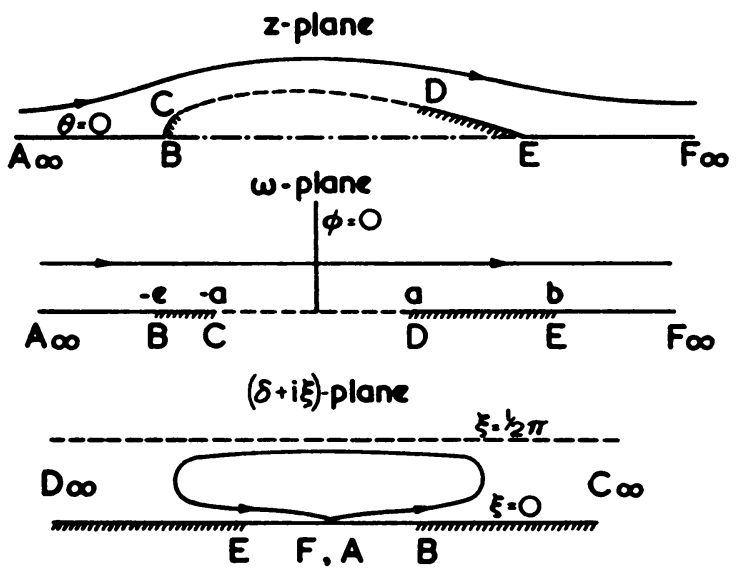

FIG.1

the velocity distribution is known, or can be deduced, as a function of $s$, the surface distance along $C D$. The equation

$$
\phi(s)=\int^{*} q d s,
$$

enables $q(\phi)$, and hence from Eq. (9), $\Omega(\phi)$, to be deduced in $-a \leq \phi \leq a$, but $\theta(\phi)$ cannot be determined exactly in $-e \leq \phi \leq-a, a \leq \phi \leq b$, until $q$ is known in these intervals. Ignoring this difficulty for the moment, we shall proceed to find the solution of Eq. (12) in the $w$-plane, with the mixed boundary conditions: $\theta(\phi)$ known in $-\infty \leq$ $\phi \leq-a, a \leq \phi \leq \infty ; \Omega(\phi)$ known in $-a \leq \phi \leq a$. 
The transformation

$$
\delta+i \xi=-\frac{1}{2} \log \left(\frac{w+a}{w-a}\right),
$$

where $\delta$ and $\xi$ are real, transforms the upper half of the $w$-plane into the infinite strip $-\infty \leq \delta \leq \infty, 0 \leq \xi \leq \frac{1}{2} \pi$. The $(\delta+i \xi)$-plane is shown in Fig. 1, and it will be noticed that in this plane the boundary conditions are particularly simple. The solution of (12) in this case has been obtained in an earlier paper [3]; it is

$f(\delta, \xi)=\frac{1}{\pi} \int_{-\infty}^{\infty}\left\{\theta\left(\delta^{*}\right) \operatorname{cosech}\left(\delta^{*}-\delta-i \xi\right)+\Omega\left(\delta^{*}\right) \operatorname{sech}\left(\delta^{*}-\delta-i \xi\right)\right\} d \delta^{*}$,

where $\theta\left(\delta^{*}\right)$ is the value of $\theta$ on $\xi=0$, and $\Omega\left(\delta^{*}\right)$ is the value of $\Omega$ on $\xi=\frac{1}{2} \pi$. Integrating (14) by parts, we find the alternative form

$$
\begin{aligned}
f(\delta, \xi)=\Omega_{c}+i \theta_{c} & +\frac{2}{\pi} \int_{\delta^{*}=-\infty}^{\infty} \tanh ^{-1} \exp \left(\delta^{*}-\delta-i \xi\right) d \theta\left(\delta^{*}\right) \\
& -\frac{2}{\pi} \int_{\delta^{*}=-\infty}^{\infty} \tan ^{-1} \exp \left(\delta^{*}-\delta-i \xi\right) d \Omega\left(\delta^{*}\right),
\end{aligned}
$$

where $\Omega_{c}$ and $\theta_{c}$ are the values of $\Omega$ and $\theta$ at $\delta=\infty$, i.e. at $\phi=-a$. From Eqs. (13) and (15)

$$
\begin{aligned}
f(w)=\Omega_{c}+i \theta_{c} & +\frac{2}{\pi}\left(\int_{\phi^{*---}}^{-a}+\int_{a}^{b}\right) \tanh ^{-1}\left\{\frac{(w+a)\left(\phi^{*}-a\right)}{(w-a)\left(\phi^{*}+a\right)}\right\}^{1 / 2} d \theta\left(\phi^{*}\right) \\
& +\frac{2}{\pi} \int_{\phi^{*}=-a}^{a} \tan ^{-1}\left\{\frac{(w+a)\left(a-\phi^{*}\right)}{(w-a)\left(a+\phi^{*}\right)}\right\}^{1 / 2} d \Omega\left(\phi^{*}\right),
\end{aligned}
$$

where, since $\theta\left(\phi^{*}\right)=0$ outside $(b,-e)$, the range of integration has been reduced. Equation (16) is the required solution of (12) in the $w$-plane.

Two important auxiliary equations follow from (16) by expanding in a power series in $(1 / w)$. It is found that

$$
\begin{aligned}
f(w) & =\left\{\Omega_{c}+\frac{2}{\pi} \int_{\phi^{*}=-e}^{-a} \tanh ^{-1}\left(\frac{\phi^{*}+a}{\phi^{*}-a}\right)^{1 / 2} d \theta\left(\phi^{*}\right)\right. \\
& \left.+\frac{2}{\pi} \int_{\phi^{*}=-a}^{a} \tan ^{-1}\left(\frac{a-\phi^{*}}{a+\phi^{*}}\right)^{1 / 2} d \Omega\left(\phi^{*}\right)+\frac{2}{\pi} \int_{\phi^{*}=a}^{b} \tanh ^{-1}\left(\frac{\phi^{*}-a}{\phi^{*}+a}\right)^{1 / 2} d \theta\left(\phi^{*}\right)\right\} . \\
& +\frac{1}{w \pi}\left\{\left(\int_{\phi^{*}--e}^{-a}+\int_{a}^{b}\right)\left(\frac{\phi^{*}-a}{\phi^{*}+a}\right)^{1 / 2}\left(\phi^{*}+a\right) d \theta\left(\phi^{*}\right)\right. \\
& \left.+\int_{\phi^{*}=-a}^{a}\left(\frac{a-\phi^{*}}{a+\phi^{*}}\right)^{1 / 2}\left(\phi^{*}+a\right) d \Omega\left(\phi^{*}\right)\right\}+0\left(1 / w^{2}\right) .
\end{aligned}
$$

Since $\theta_{\infty}=0, q_{\infty}=U$, it follows from (2) that $f_{\infty}=0$. Hence from (17)

$$
\begin{aligned}
& \Omega_{c}+\frac{2}{\pi} \int_{\phi^{*}=-e}^{-a} \tanh ^{-1}\left(\frac{\phi^{*}+a}{\phi^{*}-a}\right)^{1 / 2} d \theta\left(\phi^{*}\right)+\frac{2}{\pi} \int_{\phi^{*--a}}^{a} \tan ^{-1}\left(\frac{a-\phi^{*}}{a+\phi^{*}}\right)^{1 / 2} d \Omega\left(\phi^{*}\right) \\
&+\frac{2}{\pi} \int_{\phi^{*}=a}^{b} \tanh ^{-1}\left(\frac{\phi^{*}-a}{\phi^{*}+a}\right)^{1 / 2} d \theta\left(\phi^{*}\right)=0 .
\end{aligned}
$$


Equations (2), (17) and (18) yield

$$
\begin{aligned}
\frac{q}{U}=1-\frac{\phi}{\pi \beta_{\infty}\left(\phi^{2}+m_{\infty}^{2} \psi^{2}\right)}\left\{\left(\int_{\phi^{*}=-e}^{-a}\right.\right. & \left.+\int_{a}^{b}\right)\left(\frac{\phi^{*}-a}{\phi^{*}+a}\right)^{1 / 2}\left(\phi^{*}+a\right) d \theta\left(\phi^{*}\right) \\
& \left.+\int_{\phi^{*}=-a}^{a}\left(\frac{a-\phi^{*}}{a+\phi^{*}}\right)^{1 / 2}\left(\phi^{*}+a\right) d \Omega\left(\phi^{*}\right)\right\}+0\left(1 /|w|^{2}\right) .
\end{aligned}
$$

Since there can be no circulation about the aerofoil it follows from this result that

$$
\begin{array}{rl}
\left(\int_{0}^{-a}+\int_{a}^{b}\right)\left(\phi^{*}+a\right)\left(\frac{\phi^{*}-a}{\phi^{*}+a}\right)^{1 / 2} & d \theta\left(\phi^{*}\right) \\
& +\int_{\phi^{*}=-a}^{a}\left(\phi^{*}+a\right)\left(\frac{a-\phi^{*}}{a+\phi^{*}}\right)^{1 / 2} d \Omega\left(\phi^{*}\right)=0 .
\end{array}
$$

This equation can be regarded as a closure condition (see the closure conditions given in $[2])$.

On $\psi=0$ Eq. (16) yields

$$
\begin{aligned}
\Omega(\phi)=\Omega_{c}+R \frac{2}{\pi}\left(\int_{\phi^{*}=-e}^{-a}+\int_{a}^{b}\right) & \tanh ^{-1}\left\{\frac{\left(\phi^{*}-a\right)(\phi+a)}{\left(\phi^{*}+a\right)(\phi-a)}\right\}^{1 / 2} d \theta\left(\phi^{*}\right) \\
& +\frac{2}{\pi} \int_{\phi^{*}=-a}^{a} \tan ^{-1}\left\{\frac{\left(a-\phi^{*}\right)(\phi+a)}{\left(a+\phi^{*}\right)(\phi-a)}\right\}^{1 / 2} d \Omega\left(\phi^{*}\right),
\end{aligned}
$$

when $-e \leq \phi \leq-a, a \leq \phi \leq b$, and

$$
\begin{aligned}
\theta(\phi)=\theta_{c}-\frac{2}{\pi}\left(\int_{\phi^{*}--e}^{-a}+\int_{a}^{b}\right) & \tan ^{-1}\left\{\frac{\left(\phi^{*}-a\right)(a+\phi)}{\left(\phi^{*}+a\right)(a-\phi)}\right\}^{1 / 2} d \theta\left(\phi^{*}\right) \\
- & -Q \frac{2}{\pi} \int_{\phi^{*}--a}^{a} \tanh ^{-1}\left\{\frac{\left(a-\phi^{*}\right)(a+\phi)}{\left(a+\phi^{*}\right)(a-\phi)}\right\}^{1 / 2} d \Omega\left(\phi^{*}\right),
\end{aligned}
$$

when $-a \leq \phi \leq a$. (' $R$ ' denotes the 'real part of'.)

Two further auxiliary equations of less importance can be derived from the condition that on a well-designed aerofoil there should be no adverse infinite pressure gradients, except at the trailing edge. This requires that $\partial q / \partial s>-\infty$, or since from (1) and (2)

$$
\frac{\partial q}{\partial s}=\frac{d q}{d \Omega} \frac{\partial \Omega}{\partial \phi} \frac{\partial \phi}{\partial s}=-\frac{q^{2}}{\beta} \frac{\partial \Omega}{\partial \phi},
$$

that

$$
\frac{\partial \Omega}{\partial \phi}<\infty .
$$

Such infinite pressure gradients can occur at points separating the two types of boundary condition, i.e. at points $C$ and $D$ of Fig. 1. From (20), when $|\phi|>a$,

$$
\begin{array}{r}
\frac{\partial \Omega}{\partial \phi}=\frac{1}{\pi(\phi-a)}\left\{\frac{\phi-a}{\phi+a}\right\}^{1 / 2}\left\{\left(\int_{\phi^{*}=-e}^{-a}+\int_{a}^{b}\right)\left(\frac{\phi^{*}+a}{\phi^{*}-\phi}\right)\left(\frac{\phi^{*}-a}{\phi^{*}+a}\right)^{1 / 2} d \theta\left(\phi^{*}\right)\right. \\
\left.+\int_{\phi^{*}=-a}^{a}\left(\frac{\phi^{*}+a}{\phi^{*}-\phi}\right)\left(\frac{a-\phi^{*}}{a+\phi^{*}}\right)^{1 / 2} d \Omega\left(\phi^{*}\right)\right\},
\end{array}
$$


so that if (22) is to hold when $\phi=a$, and when $\phi=-a$, we must have

and

$$
\left(\int_{\phi^{*}=-\infty}^{-a}+\int_{a}^{b}\right)\left(\frac{\phi^{*}+a}{\phi^{*}-a}\right)^{1 / 2} d \theta\left(\phi^{*}\right)-\int_{\phi^{*}-a}^{a}\left(\frac{a+\phi^{*}}{a-\phi^{*}}\right)^{1 / 2} d \Omega\left(\phi^{*}\right) \leq 0,
$$

$$
\left(\int_{\phi^{*}--e}^{-a}+\int_{a}^{b}\right)\left(\frac{\phi^{*}-a}{\phi^{*}+a}\right)^{1 / 2} d \theta\left(\phi^{*}\right)+\int_{\phi^{*}=-a}^{a}\left(\frac{a-\phi^{*}}{a+\phi^{*}}\right)^{1 / 2} d \Omega\left(\phi^{*}\right) \geq 0,
$$

respectively. This completes the mathematical theory.

3. Method of designing an aerofoil. For a rounded-nose aerofoil the discontinuity in $\theta$ at the leading edge, $\phi=-e$, is $\frac{1}{2} \pi$, while at the trailing edge, $\phi=b$, it is $\frac{1}{2} \tau$, where $\tau$ is thus the trailing-edge angle. If for simplicity we assume that no other discontinuities in $\theta$ exist, then in $-e<\phi \leq-a$, and $a \leq \phi<b$, we can write for the value of $d \theta\left(\phi^{*}\right)$ occurring in Eqs. (18)-(21)

$$
d \theta\left(\phi^{*}\right)=\frac{d \theta}{d s} \frac{\partial s}{\partial \phi^{*}} d \phi^{*}=-\frac{d \phi^{*}}{R q}
$$

where $R$ is the radius of curvature of the surface.

Equation (21) can be regarded as the 'design equation' as it determines the aerofoil shape in the interval where the pressures are specified. However before it can be used for this purpose, it is clear from (25) that it is necessary to know $q(\phi)$ in $-e<\phi \leq-a$, and $a \leq \phi<b$, and this can only be found by first solving Eq. (20). It is apparent from (9) and (25) that (20) is an integral equation for $q$. It can be solved by iteration as follows. We assume some likely distribution $q(\phi)$; use it in

$$
s(\phi)=\int^{\phi} \frac{d \phi}{q}
$$

to find $s(\phi)$, and hence from the known $R(s)$, to find $R(\phi)$. These values of $R(\phi)$ and $q(\phi)$ are then used in (25), (18) and (20) to deduce $\Omega(\phi)$. The first iteration is completed by calculating a new $q(\phi)$ from (9). When these iterations have converged $\dagger$, the final values of $R(\phi)$ and $q(\phi)$ are used in (21) and (25) to complete, without further iteration, the design of the aerofoil. Equation (19) can be conveniently satisfied by varying one of the parameters $e, a$ or $b$ during the iterative process. Finally the aerofoil co-ordinates follow from

$$
x=\int^{\phi} \frac{\cos \theta d \phi}{q}, \quad y=\int^{\phi} \frac{\sin \theta d \phi}{q} .
$$

4. Special cases. (a) Flow about a given symmetrical aerofoil.

From Fig. 1 it is apparent that the solution of this problem is obtained by letting ' $a$ ' tend to zero. In this case Eqs. (18) and (19) yield

$$
\int_{\phi^{*}--\infty}^{b} d \theta\left(\phi^{*}\right)=0, \quad \int_{\phi^{*}=-e}^{b} \phi^{*} d \theta\left(\phi^{*}\right)=0 .
$$

Subtracting (18) from (16), taking the limit as ' $a$ ' tends to zero, and making some use of (28) we find

$$
f(w)=-\frac{1}{\pi} \int_{\phi^{*}=-\infty}^{b} \log \left(\phi^{*}-w\right) d \theta\left(\phi^{*}\right) .
$$

$\dagger$ Experience with the same type of integral equation leads the author to believe that practical convergence is almost certain. 
These three equations are the basis of the author's method [4] of calculating the flow about given symmetrical aerofoils.

(b) Usual equation of aerofoil design. This is arrived at by putting $e, b=a$ in the above theory. If we write $\phi^{*}=-a \cos \gamma^{*}$. Then (18) and (19) become (see [1] and [2])

$$
\int_{0}^{\pi} \Omega\left(\gamma^{*}\right) d \gamma^{*}=0, \quad \int_{1}^{\pi} \Omega\left(\gamma^{*}\right) \cos \gamma^{*} d \gamma^{*}=0,
$$

while on the aerofoil surface $\mathrm{E}_{(1}$. (16) reduces to the design equation [2]

$$
\theta(\phi)=-\frac{1}{2 \pi} \int_{0}^{\pi} \Omega\left(\gamma^{*}\right)\left\{\cot \frac{1}{2}\left(\gamma^{*}-\gamma\right)-\cot \frac{1}{2}\left(\gamma^{*}+\gamma\right)\right\} d \gamma^{*} .
$$

(c) Riabouchinsky flow. Riabouchinsky flow [5] may be defined as flow past two solid bodies between which there is a bubble or cavity at constant pressure. All the steady symmetrical Riabouchinsky flows can be calculated from equations (16), (18) and (19) by putting $d \Omega\left(\phi^{*}\right)=0$. (Unsteady Riabouchinsky flow is discussed in British A.R.C. Current Paper No. 149.)

(d) A simple family of 'roof-top' aerofoils. 'Roof-top' aerofoils are those for which the specified velocity distribution is composed of two linear segments. Such aerofoils can be designed by putting $d \Omega\left(\phi^{*}\right)=k_{1},(-a \leq \phi \leq h),=k_{2},(h \leq \phi \leq a)$, where $k_{1}$ and $k_{2}$ are constants, in Eqs. (18)-(21).

For the simple example which has $k_{1}=k_{2}=0$, and $\theta$ constant in $-e \leq \phi \leq-a$, $a \leq \phi \leq b$, except for discontinuities of $\frac{1}{2} \pi$ and $\frac{1}{2} \tau$ at the leading and trailing edges respectively, these equations yield

$$
\begin{gathered}
\Omega_{c}=-\tanh ^{-1}\left(\frac{e-1}{e+1}\right)^{1 / 2}-\frac{\tau}{\pi} \tanh ^{-1}\left(\frac{b-1}{b+1}\right)^{1 / 2}, \\
\frac{\tau}{\pi}=\left(\frac{e^{2}-1}{b^{2}-1}\right)^{1 / 2}, \\
\theta(\phi)=\frac{1}{2} \pi-\tan ^{-1}\left\{\frac{(e+1)(1+\phi)}{(e-1)(1-\phi)}\right\}^{1 / 2}-\frac{\tau}{\pi} \tan ^{-1}\left\{\frac{(b-1)(1+\phi)}{(b+1)(1-\phi)}\right\}^{1 / 2},|\phi|<1,
\end{gathered}
$$

and

$$
\begin{aligned}
& \Omega(\phi)=\Omega_{c}+Q \tanh ^{-1}\left\{\frac{(e+1)(\phi+1)}{(e-1)(\phi-1)}\right\}^{1 / 2} \\
& \quad+\frac{\tau}{\pi} R \tanh ^{-1}\left\{\frac{(b-1)(\phi+1)}{(b+1)(\phi-1)}\right\}^{1 / 2}, \quad|\phi|>1,
\end{aligned}
$$

where ' $a$ ' has been put equal to unity without loss of generality. With the aid of (29) we find that the left hand sides of (23) and (24) reduce to $1 /(e+1)+1 /(b-1)$, and $1 /(e-1)+1 /(b+1)$, respectively. Thus, since $e, b>1$, it follows from (23) and (24) that while an infinite adverse pressure gradient does not occur at $\phi=-1$, it is unavoidable at $\phi=1$.

Typical values of $\tau$ and $b$ for a modern aerofoil would be $\tau=12^{\circ}$, and $b=2.5$, and from (29) and (28), $e=1.0117$ and $\Omega_{c}=-0.1287$. Suppose $M_{\infty}=0.7$, then from Eqs. (9) and (10) we find that the constant velocity of the roof-top is $q=1.22 U$. We shall omit further details of this example, but it should be noted that the flat nose occupies 
less than $0.4 \%$ of the total potential difference over the chord, so that it could be roundedoff with but little effect on the velocity distribution. Eqs. (28)-(31) thus provide a comparatively quick method of designing roof-top aerofoils.

An alternative method of design would be to let $e$ tend to ' $a$ ' and then specify the velocity distribution in the interval between the nose and the beginning of the flat section of the velocity distributions so as to obtain the desired nose-radius of curvature [2].

\section{REFERENCES}

1. M. J. Lighthill, A new method of two-dimensional aerodynamic design, British Aero. Res. Council R. \& M. 2112 (1945)

2. L. C. Woods, Aerofoil design in two-dimensional subsonic flow, British Aero. Res. Council R. \& M. 2845 (1952)

3. L. C. Woods, Compressible subsonic flow in two-dimensional channels with mixed boundary conditions, to be published shortly in the Quart. J. Mech. and Appl. Math.

4. L. C. Woods, The application of the polygon method to the calculation of the compressible subsonic flow round two-dimensional profiles, British Aero. Res. Council Current Paper No. 115 (1952)

5. G. Birkhoff, Hydrodynamics: A study in logic, fact and similitude, Princeton University Press, 1950

6. Th. von Kármán, Compressibility effect in aerodynamics, J. Aero. Sci. 8, No. 9 (1941) 